\title{
New Insights into the Effects of Several Environmental Parameters on the Relative Fitness of a Numerically Dominant Class of Evolved Niche Specialist
}

\author{
Anna Kuśmierska ${ }^{1,2}$ and Andrew J. Spiers ${ }^{1}$ \\ ${ }^{1}$ School of Science, Engineering and Technology, Abertay University, Bell Street, Dundee DD1 1HG, UK \\ ${ }^{2}$ Department of Industrial Microbiology and Biotechnology, Faculty of Biology and Environmental Protection, \\ University of Łódź, Łódź, Poland
}

Correspondence should be addressed to Andrew J.Spiers; a.spiers@abertay.ac.uk

Received 30 September 2016; Accepted 24 November 2016

Academic Editor: Santiago F. Elena

Copyright (C) 2016 A. Kuśmierska and A. J. Spiers. This is an open access article distributed under the Creative Commons Attribution License, which permits unrestricted use, distribution, and reproduction in any medium, provided the original work is properly cited.

\begin{abstract}
Adaptive radiation in bacteria has been investigated using Wrinkly Spreaders (WS), a morphotype which colonises the air-liquid (A-L) interface of static microcosms by biofilm formation with a significant fitness advantage over competitors growing lower down in the $\mathrm{O}_{2}$-limited liquid column. Here, we investigate several environmental parameters which impact the ecological opportunity that the Wrinkly Spreaders exploit in this model system. Manipulation of surface area/volume ratios suggests that the size of the WS niche was not as important as the ability to dominate the A-L interface and restrict competitor growth. The value of this niche to the Wrinkly Spreaders, as determined by competitive fitness assays, was found to increase as $\mathrm{O}_{2}$ flux to the A-L interface was reduced, confirming that competition for $\mathrm{O}_{2}$ was the main driver of WS fitness. The effect of $\mathrm{O}_{2}$ on fitness was also found to be dependent on the availability of nutrients, reflecting the need to take up both for optimal growth. Finally, the meniscus trap, a high- $\mathrm{O}_{2}$ region formed by the interaction of the A-L interface with the vial walls, was also important for fitness during the early stages of biofilm formation. These findings reveal the complexity of this seemingly simple model system and illustrate how changes in environmental physicality alter ecological opportunity and the fitness of the adaptive morphotype.
\end{abstract}

\section{Introduction}

Adaptive radiation requires organismal evolvability, ecological opportunities, and diversifying selection which create new niches in terms of physical space and new interactions between organisms and environment or allow empty ones to be colonised by adaptive lineages [1-4]. This process is directly relevant to the bacterial colonisation of natural and engineered environments, affecting final population sizes (productivity) and other ecosystem processes, host survival, efficiency, and output, and can be investigated in experimental evolution studies using simple microcosms in which biotic and abiotic factors and parameters can be manipulated [5-8].

One particularly successful system has used Pseudomonas fluorescens SBW25 populations grown in small glass vials containing nutritionally rich King's B medium [9]. These microcosms can be incubated statically to produce a heterogeneous environment with spatial structure and, in these, $P$. fluorescens SBW25 populations rapidly diversify over 3-10 days and accumulate mutants such as the Wrinkly Spreader. This class of adaptive mutant or morphotype [8] produces distinctive wrinkled colonies on agar plates and a robust, well-attached biofilm at the air-liquid (A-L) interface of static microcosms (sometimes referred to as pellicles, but see [10]) (Figure 1). It is the ecosystem engineering [3] by the early $P$. fluorescens SBW25 colonists that generates an $\mathrm{O}_{2}$ gradient in these microcosms and produces the ecological opportunity for Wrinkly Spreader colonisation of the AL interface [11] (opportunity and niche are interlinked; see $[8,12,13])$. A range of mutations associated with diguanylate cyclases (DGCs) result in the Wrinkly Spreader (WS) phenotype through upregulation of $c-d i$-GMP levels and the 


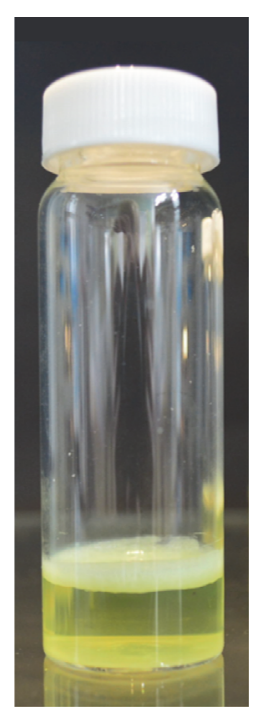

(a)

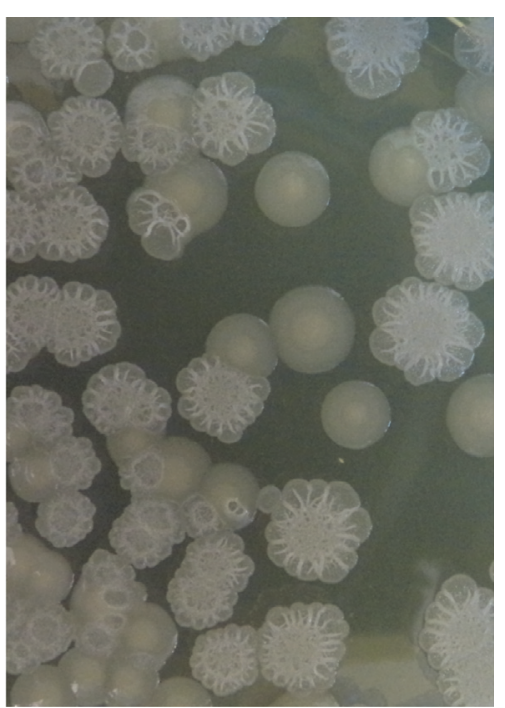

(b)

FIGURE 1: The Wrinkly Spreader is an adaptive morphotype which colonises the A-L interface in static microcosms. The WS phenotype is defined by (a) the formation of a robust, well-attached biofilm in static microcosms and (b) wrinkled colonies on agar plates which are readily distinguished from the smooth, rounded colonies produced by wild-type P. fluorescens SBW25.

overproduction of partially acetylated cellulose (the primary extracellular polymeric substance (EPS) or matrix component) and attachment factor essential for colony morphology and biofilm structure [14-18]. This influential but simple experimental evolution model has been used to investigate aspects of adaptive radiation, including the importance of spatial structure (with high- and low- $\mathrm{O}_{2}$ zones) and resource competition (for $\mathrm{O}_{2}$ and nutrients), and of the emergence and maintenance of diversity $[6,8]$ which can be readily measured by determining the frequencies and final population sizes (productivity) of Wrinkly Spreaders and other morphotypes on agar plates (e.g., $[9,19,20])$.

Our interests are more focussed on developing a mechanistic explanation of how environmental parameters influence Wrinkly Spreader biofilm formation and fitness in this simple microcosm system. Colonisation of the A-L interface by the Wrinkly Spreader allows better access to $\mathrm{O}_{2}$ diffusing into the liquid column from the air above, providing a competitive fitness $(W)$ advantage over the ancestral wildtype $P$. fluorescens SBW25 and other non-biofilm-forming mutants whose growth is $\mathrm{O}_{2}$-limited deeper into the microcosm $[9,11,14,21]$. This advantage may reflect a significant change of physiology, as biofilm-isolated cells growing in the high- $\mathrm{O}_{2}$ zone can be differentiated from those recovered immediately below the biofilm by Raman spectral profiling [22] (P. fluorescens SBW25 can form a different type of biofilm when induced with $\mathrm{Fe}^{3+}$ which also provides a fitness advantage [23]). In the related pseudomonad, $P$. aeruginosa PA01, cells grow aerobically in A-L interface biofilms and, like the Wrinkly Spreader, these have a growth advantage over non-biofilm-forming competitors which are $\mathrm{O}_{2}$-stressed [24]. Furthermore, EPS production may be altruistic to cells as it pushes later generations into better $\mathrm{O}_{2}$ conditions above and helps suffocate non-EPS producers and cells lower down in the biofilm (ancestor's inhibition) [25]. $\mathrm{O}_{2}$ levels and the lack of physical disturbance (i.e., random knocks and vibrations) are probably the most important factors driving Wrinkly Spreader biofilm formation and longevity [11]. However, the advantage the Wrinkly Spreader has in static microcosms does not translate to other environments, as it is at a disadvantage in shaken microcosms and on agar plates where the WS phenotype is irrelevant and costly, and the ancestor effect may not be effective $[9,14,21,26]$.

As Wrinkly Spreaders are an adaptive morphotype in static microcosms (and because ecological opportunity and niche are interlinked), we expect that a "Goldilocks" effect should be apparent in these simple microcosms, and if environmental parameters differed, perhaps the Wrinkly Spreaders would not have the competitive fitness advantage they have over non-biofilm-forming competitors. In particular, there should be a tight link between the potential growth achievable at the A-L interface and WS fitness. However, the role of $\mathrm{O}_{2}$ is complex as the amount available, considered in terms of local concentration, during biofilm formation provides the ecological opportunity and reward for WS colonisation of the A-L interface, but $\mathrm{O}_{2}$ flux or supply (determined by the diffusion from the air above and uptake by the bacteria in the liquid column below) may have a more immediate impact on the developing WS biofilm and competitors. Furthermore, the importance of $\mathrm{O}_{2}$ also depends on nutrient levels and other factors, as if these became growth-limiting, $\mathrm{O}_{2}$ would no longer be a reward for colonisation of the A-L interface.

In this work, we manipulate several environmental parameters predicted to alter $\mathrm{O}_{2}$ levels in static microcosms in order to investigate how competition for this growth-limiting resource affects WS fitness in more detail and to further our understanding of the mechanisms underlying this commonly used model system. 


\section{Materials and Methods}

2.1. Bacteria and Culture Conditions. The bacterial strains used in this work were wild-type Pseudomonas fluorescens SBW25 [27] and the archetypal Wrinkly Spreader ( $P$. fluorescens SBW25 wspF A901C) [14,17]. Bacteria were cultured at $18-20^{\circ} \mathrm{C}$ in modified King's B $\left(\mathrm{KB}^{*}\right)$ medium $(20 \mathrm{~g}$ proteose peptone (Oxoid, UK), $10 \mathrm{~g}$ glycerol, $1.5 \mathrm{~g} \mathrm{~K}_{2} \mathrm{HPO}_{4}$, and $1.5 \mathrm{~g}$ $\mathrm{MgSO}_{4}$ per litre with $1.5 \%(\mathrm{w} / \mathrm{v})$ agar added for plates) and maintained at $-80^{\circ} \mathrm{C}$ as $15 \%(\mathrm{w} / \mathrm{v})$ glycerol stocks. The quantities of peptone and glycerol were reduced appropriately to produce media with $0.1 \mathrm{x}$ and $0.01 \mathrm{x}$ normal levels of nutrients. Standard microcosms were $30 \mathrm{ml}$ Universal glass vials containing $10 \mathrm{ml} \mathrm{KB}{ }^{*}$ and were incubated statically or with shaking at $150 \mathrm{rpm}$ using a Stuart S150 orbital incubator (Bibby Scientific Ltd., UK). Mineral oil (Fisher BioReagents, UK) overlays of $10-40 \mathrm{~mm}$ were added to microcosms to reduce $\mathrm{O}_{2}$ flux. Glass conical flasks and test tubes with $2.5-15 \mathrm{ml} \mathrm{KB}{ }^{*}$ were used to produce microcosms with different surface area/volume ratios. A $14 \mathrm{~cm}$ diameter Petri dish containing $100 \mathrm{ml} \mathrm{KB}^{*}$ was used to assess biofilm formation over larger surfaces. Universal vials were filled to the brim with $37 \mathrm{ml}$ and $39.5 \mathrm{ml} \mathrm{KB}^{*}$ to produce microcosms with concave- (normal) and convex-shaped A-L interfaces, respectively. In order to minimise evaporation during the convex-concave experiments, microcosms were incubated in sealed containers with a dish of water to maintain humidity, and fresh medium was added every $\sim 12 \mathrm{hr}$ to maintain the convex shape (equal additions were also made to the concave microcosms).

2.2. Spectroscopy. A Spectronic Helios Epsilon spectrophotometer (Thermo Fisher Scientific, UK) was used for absorbance and optical density measurements using $1 \mathrm{~cm}$ optical-pathway cuvettes after zeroing using the appropriate sterile growth medium or solvent.

2.3. Preliminary Growth, Biofilm, and Oil Toxicity Tests. Preliminary tests were made to determine whether reducednutrient or oil-overlay microcosms impacted $P$. fluorescens SBW25 growth and WS biofilm formation. Microcosms were inoculated with $100 \mu \mathrm{l}$ aliquots of overnight static WS or shaken WT cultures as appropriate. Growth differences were assessed by optical density $\left(\mathrm{OD}_{600}\right)$ measurements after $24 \mathrm{hr}$ incubation. WS biofilm formation was assessed visually after 3 days. Oil toxicity was tested by comparing growth $\left(\mathrm{OD}_{600}\right)$ between shaken $\mathrm{KB}^{*}$ microcosms with and without $100 \mu \mathrm{l}$ oil after $24 \mathrm{hr}$ incubation. For these, microcosms were left to stand for $30 \mathrm{~min}$ after vigorous mixing to avoid the transfer of emulsion or oil to the sample used for $\mathrm{OD}_{600}$ measurement.

2.4. Competitive Fitness Assay. The competitive fitness of the Wrinkly Spreader (WS) was determined relative to wildtype P. fluorescens SBW25 (WT) [21]. Replicate microcosms were inoculated with $100 \mu \mathrm{l}$ (per $10 \mathrm{ml}$ ) aliquots of a $1: 1$ mixture of overnight static WS and shaken WT cultures and incubated for 3 days before assay. The initial and final WS and WT viable cell numbers were determined by sampling the 1:1 mixture and 3-day microcosms after vigorous mixing, serial dilution, and enumeration on $\mathrm{KB}^{*}$ plates. Competitive fitness $(W)$ was calculated as the mean $W=$ $\ln$ [final WS/initial WS]/ $\ln$ [final WT/initial WT] [28]. Relative fitness $\left(W / W_{\mathrm{R}}\right)$ is reported where $\mathrm{R}$ refers to the reference microcosms used for each assay.

2.5. Combined Biofilm Assay. Replicate microcosms $(n=8)$ were inoculated with $100 \mu \mathrm{l}$ (per $10 \mathrm{ml}$ ) aliquots of overnight static Wrinkly Spreader culture and incubated for 3 days before sequential assay to determine growth, biofilm strength, and attachment levels [29]. Briefly, biofilm strength (S) was first measured using the maximum deformation mass (grams) assay with small glass balls [30]. The microcosm contents were then transferred to another vial and, after vigorous mixing, used to determine growth $(G)$ by optical density $\left(\mathrm{OD}_{600}\right)$ measurements. The empty vial was then stained with Crystal Violet and the absorbance $\left(\mathrm{A}_{570}\right)$ measurements of the eluted dye were used to determine the attachment levels (A) in the meniscus region [15]. It was necessary to wipe the outside rim of the vials to remove excess stain from the concave and convex microcosms before elution and measurement. Relative growth, biofilm strength, and attachment levels were calculated as for relative fitness (e.g., $G / G_{R}$, where $\mathrm{R}$ refers to the reference microcosms used in that assay).

2.6. Statistical Analyses. Data were analysed using JMP 12 statistical software (SAS Institute Inc., USA) and means with standard errors (SE) are reported. Normal quantile plots of the residuals were inspected with outliers removed if required and normality was assessed using the Shapiro-Wilk $\mathrm{W}$ goodness-of-fit test $(p>0.05)$. In large experiments, individual treatments were processed as single batches and, as a result, batch and treatment effects are combined. Comparison of means was by $t$-test and ANOVA, with post hoc comparisons made by Dunnett's test with control and TukeyKramer HSD tests.

\section{Results and Discussion}

3.1. The Experimental Microcosm System and the Determination of Fitness. As WS fitness is negatively frequency dependent [9], competition between Wrinkly Spreaders and the non-biofilm-forming $P$. fluorescens SBW25 competitor used in this work will vary depending on the relative starting ratio of strains. When the Wrinkly Spreader is rare, competition will primarily occur between the two strains and WS fitness will be high, but as the Wrinkly Spreaders become dominant, within-WS competition will increase and WS fitness will fall. In this work, we have balanced experimental workloads with the range of environmental parameters that could be investigated. We have chosen test WS fitness using a $1: 1$ starting ratio of strains at a standard cell density from which the Wrinkly Spreaders are expected to become rapidly dominant and produce a biofilm when conditions are favourable. Under similar starting conditions, changes in WS biofilm characteristics can be quantified $[15,29,30]$, giving us confidence in linking WS fitness with biofilm formation. However, we note that alterations in these starting conditions will alter the dynamics of the system and possibly final outcomes. Finally, as in many of 
our experiments we observed relatively small fitness changes, we have chosen to report relative fitness $\left(W / W_{\mathrm{R}}\right)$ using the appropriate reference microcosms, to reflect the magnitude of change rather than absolute competitive fitness $(W)$ values.

3.2. WS Fitness Depends on the Ability to Dominate the A$L$ Interface and Reduce $\mathrm{O}_{2}$ Availability for Competitors. We predict that the competitive success of the Wrinkly Spreader in colonising the A-L interface of static microcosms is influenced by the physical size of the niche space available for WS colonisation compared to that available to the competitor. WS biofilms develop across the A-L interface before developing in depth and strength [15]. This suggests that the residents of the WS biofilm benefit by the biofilm spreading out across the A-L interface first to intercept $\mathrm{O}_{2}$ diffusion into the liquid column, and their growth subsequently becomes limited by reduced $\mathrm{O}_{2}$ and nutrient diffusion into the biofilm as it matures and thickens. As this area expansion-first strategy also has the effect of limiting $\mathrm{O}_{2}$ diffusion lower down into the liquid column, we do not expect that non-biofilm-forming competitors would be advantaged by deeper microcosms with greater nutrient resources as growth will still be $\mathrm{O}_{2}$ limited. We therefore expect to find that WS fitness will fall in microcosms with smaller A-L interface surface areas (SA) and fixed volumes $(\mathrm{V})$ but will remain unchanged in microcosms with a fixed SA and increasing V.

We used a series of flasks, vials, and test tubes to produce microcosms with a range of $\mathrm{SA} / \mathrm{V}$ ratios but with a fixed volume, from 0.15 to $1.02 \mathrm{~cm}^{-1}$. As the $\mathrm{SA}$ was reduced relative to $\mathrm{V}$ in these, the physical dimensions of the niche available for WS colonisation fell, and a small but significant decrease in relative fitness to $0.944 \pm 0.006\left(W / W_{\mathrm{R}}\right)$ was observed in the microcosms with a SA/V ratio of $0.15 \mathrm{~cm}^{-1}$ compared to the reference microcosms with a $\mathrm{SA} / \mathrm{V}$ ratio of $0.45 \mathrm{~cm}^{-1}$, though not between the microcosms with SA/V ratios of $0.45 \mathrm{~cm}^{-1}$ and $1.02 \mathrm{~cm}^{-1}$ (TK-HSD, $\alpha=0.05$ ) (Figure 2). However, no significant differences in relative fitness were observed in fixed SA microcosms containing $2.5-15 \mathrm{ml}$ $\mathrm{KB}^{*}$ (TK-HSD, $\alpha=0.05$ ). These findings suggest that competitive interaction between the Wrinkly Spreaders and the non-biofilm-forming competitor is an inhibitory one that involves the Wrinkly Spreaders preventing competitors access to $\mathrm{O}_{2}$ which slows their growth and reduces maximal population size. We note that the Wrinkly Spreader can produce a biofilm in a $14 \mathrm{~cm}$ diameter container within three days, suggesting that biofilm formation is not limited by the need to be in close proximity to the vial walls. However, Wrinkly Spreaders may also have a maximal population size; as Wrinkly Spreader numbers grow in the maturing biofilm, only the top $\sim 300 \mu \mathrm{m}$ layer of the biofilm remains $\mathrm{O}_{2}$-rich and lower regions of the biofilm become increasingly $\mathrm{O}_{2}$-limited [11]. In this situation, Wrinkly Spreaders start to compete with one another within the biofilm itself, with different WS mutants showing substantial morphological, metabolic, and fitness variation $[17,31-33]$.

We are, however, surprised that in deep microcosms the volume of media beneath the biofilm does not become increasingly attractive for colonisation by wild-type $P$. fluorescens SBW25 or a low- $\mathrm{O}_{2}$-adapted mutant, as we assume

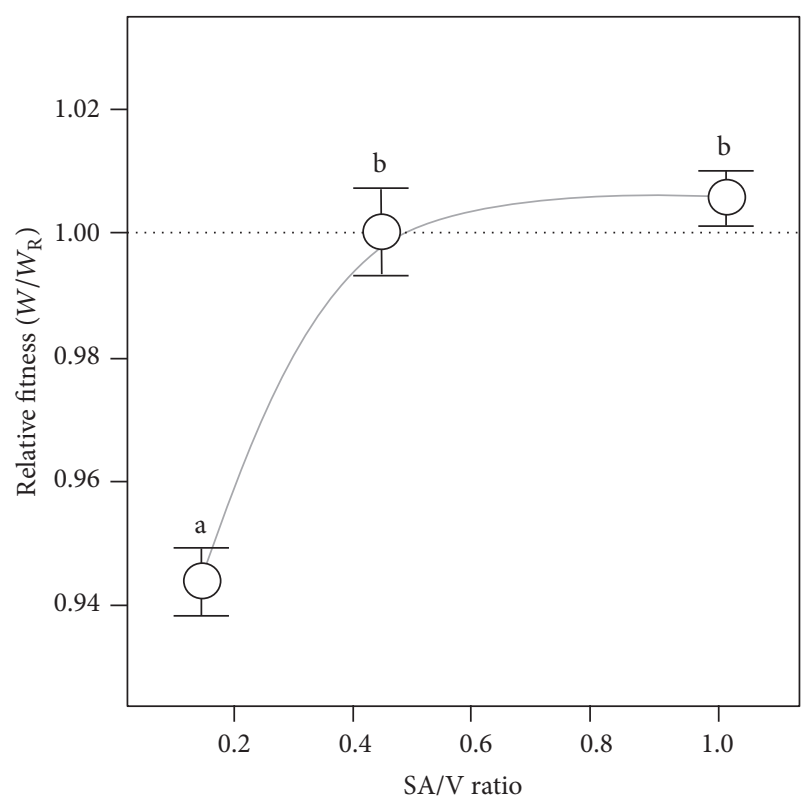

FIGURE 2: Expanding the physical niche available for colonisation increases Wrinkly Spreader fitness. Differently shaped containers were used to produce microcosms with a range of surface areas but with constant volumes (shown here as the SA/V ratio). Microcosms were incubated for 3 days before assay and the competitive fitness $(W)$ of the Wrinkly Spreader was determined compared to the non-biofilm-forming $P$. fluorescens SBW25. Here, the relative fitness $\left(W / W_{\mathrm{R}}\right)$ is provided where the reference microcosms with a $\mathrm{SA} / \mathrm{V}$ ratio of 0.45 have a relative fitness of one (marked by the horizontal dashed line); the curve indicated here is illustrative only. Means \pm SE are shown $(n=6)$, and means not linked by the same letter are significantly different (TK-HSD, $\alpha=0.05$ ).

that this volume represents an ecological opportunity for an appropriately adaptive strain, as much as the A-L interface does for the Wrinkly Spreaders. Early work had suggested that the Fuzzy Spreader might be a bottom dweller [9], but this class of mutant has subsequently been shown to be a failed biofilm former which forms a sediment after physical disturbance [34]. In the case of $P$. aeruginosa PA01, supplementation with the alternative electron-acceptor nitrate facilitated the anaerobic growth of non-biofilm-forming competitors, suggesting that cells growing in the A-L interface biofilm face a trade-off between $\mathrm{O}_{2}$ and nutrient acquisition [24]. Similarly, in Komagataeibacter xylinus (formerly Acetobacter xylinum) A-L interface biofilms, growth is limited to a top layer of $50-100 \mu \mathrm{m}$ by $\mathrm{O}_{2}$ diffusion from above and nutrient diffusion from below [35]. Although $P$. fluorescens SBW25 is regarded by some as an obligate aerobe [34], this has yet to be established and we have not yet tried supplementing $\mathrm{KB}^{*}$ with nitrate (or nitrite) to investigate the impact this would have on WS fitness or the colonisation of the low- $\mathrm{O}_{2}$ region of our microcosms.

3.3. Niche Quality Is Also Sensitive to $\mathrm{O}_{2}$ Flux. Earlier work had shown that $\mathrm{O}_{2}$ availability, considered in terms of local concentration, affected WS competitive fitness as WS competitive fitness $(W)$ fell from 1.23 under normal $\mathrm{O}_{2}$ conditions 
to 0.12 when $\mathrm{O}_{2}$ levels were reduced to $\sim 0.05 \%$ of normal levels [11]. However, $\mathrm{O}_{2}$ flux or supply (measured in terms of quantity/time/area) to the A-L interface is also likely to be a significant factor contributing to resource scarcity. Flux to the thin layer of liquid at the A-L interface where the WS biofilm is formed (the physical niche space) is dependent on the diffusion rate of $\mathrm{O}_{2}$ through air and water and on the uptake of $\mathrm{O}_{2}$ by bacteria at the A-L interface and lower down in the liquid column. Whilst flux differences affecting local $\mathrm{O}_{2}$ concentrations might alter growth rates, the total amount of $\mathrm{O}_{2}$ made available in these two regions will determine maximal population sizes over the 1-3 days in which WS biofilms form and our fitness assays are undertaken.

We have used mineral oil overlays to reduce the diffusion of $\mathrm{O}_{2}$ to the oil-aqueous interface to investigate how changes in flux might affect WS fitness. An oil layer lying between the air and the $\mathrm{KB}^{*}$ liquid column represents a diffusivity barrier to $\mathrm{O}_{2}$, as diffusion in light oil is lower than that in water and air (approx. $1 \times 10^{-9}, 2 \times 10^{-5}$, and $2 \times 10^{-1} \mathrm{~cm}^{2} / \mathrm{s}$, resp., at $25-37^{\circ} \mathrm{C}$ ) [36]. As a result, $\mathrm{O}_{2}$ flux or supply to the top of the $\mathrm{KB}^{*}$ liquid column will be lowered by an intervening oil layer in an inverse proportional depth manner once the bacteria begin to take up $\mathrm{O}_{2}$. Preliminary experiments showed that the mineral oil used here had no toxic effect on $P$. fluorescens SBW25 growth in shaken microcosms (one-tailed $t$-test, $p=0.9993$ ) and that WS biofilms formed immediately below the oil layer at the oil-aqueous interface of static microcosms containing $10-40 \mathrm{~mm}$ oil. However, although WS biofilms were produced in these oil-overlay microcosms, they were significantly reduced in terms of growth, strength, and attachment levels compared to the reference microcosms without oil (Table 1), suggesting that Wrinkly Spreader colonisation of the oil-aqueous interface was less successful. However, a small but significant increase in relative fitness up to $1.096 \pm 0.008\left(W / W_{\mathrm{R}}\right)$ was observed in microcosms with 20 and $40 \mathrm{~mm}$ oil overlays compared to the reference microcosms with no oil (TK-HSD, $\alpha=0.05$ ) (Figure 3), suggesting that the value of colonising the oilaqueous interface increased with reduced $\mathrm{O}_{2}$ flux.

Although we initially struggled to resolve the apparent conflict between our oil-overlay fitness results with that of earlier work [11], we can propose a model which links both experimental results through an understanding of how local $\mathrm{O}_{2}$ concentrations and $\mathrm{O}_{2}$ flux to the A-L interface influence WS biofilm formation and fitness. At very low $\mathrm{O}_{2}$ levels and negligible flux, as provided by the sealed anaerobic bags used in the earlier experiments [11], the cost of biofilm formation by the Wrinkly Spreader was higher than the limited and short-term growth advantage achieved under these conditions, and, as a result, the Wrinkly Spreader had no fitness advantage over the non-biofilm-forming competitor (we calculate relative fitness of $0.12\left(W / W_{\mathrm{R}}\right)$ in the low$\mathrm{O}_{2}$ conditions compared to normal $\mathrm{O}_{2}$ levels from [11]). In contrast, although $\mathrm{O}_{2}$ flux was reduced in our oil-overlay experiments and impacted the growth and maximal population sizes of both the Wrinkly Spreader and the competitor, local $\mathrm{O}_{2}$ concentrations and the continued supply of $\mathrm{O}_{2}$ from the atmosphere allowed further growth and provided the ecological reward for WS biofilm formation. We might

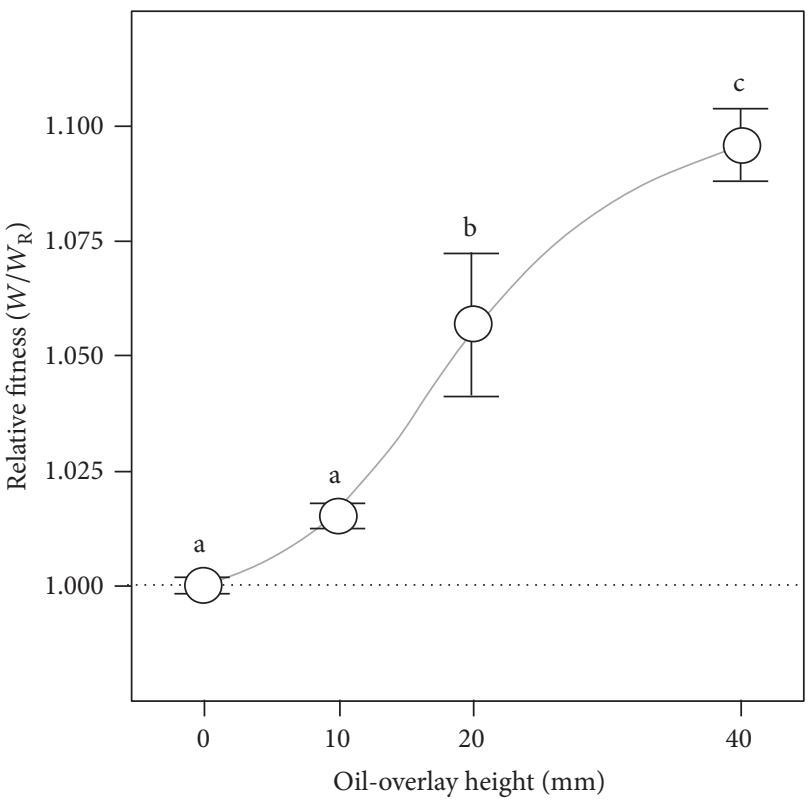

FIGURE 3: Reducing the quality of the niche available for colonisation by lowering $\mathrm{O}_{2}$ flux reduces Wrinkly Spreader fitness. Oil overlays of 10,20 , and $40 \mathrm{~mm}$ were used to reduce $\mathrm{O}_{2}$ flux or supply to the A-L or oil-KB* interface in static microcosms. Microcosms were incubated for 3 days before assay and the relative competitive fitness of the Wrinkly Spreader was determined compared to the non-biofilmforming $P$. fluorescens SBW25. Here, the relative fitness $\left(W / W_{\mathrm{R}}\right)$ is provided where the reference microcosms with no oil have relative fitness of one (marked by the horizontal dashed line); the curve indicated here is illustrative only. Means \pm SE are shown $(n=6)$, and means not linked by the same letter are significantly different (TK-HSD, $\alpha=0.05$ ). The standard $\mathrm{KB}^{*}$ microcosm (no oil) data are also shown in Figure 3.

expect that if a higher-density starting inoculum was used, sufficient $\mathrm{O}_{2}$ might be removed from the $\mathrm{KB}^{*}$ liquid column such that the reduced $\mathrm{O}_{2}$ flux could never supply sufficient additional $\mathrm{O}_{2}$ to allow the development of a biofilm. Similarly, a shorter incubation period should reduce WS performance as we expect that the maximum growth in Wrinkly Spreader populations occurs once the biofilm has been established in the high- $\mathrm{O}_{2}$ region of the microcosm, whilst longer incubation with a sufficiently high- $\mathrm{O}_{2}$ flux should improve matters whilst the biofilm develops in size and until withinWS competition begins to dominate.

3.4. The Importance of $\mathrm{O}_{2}$ to WS Fitness Is Linked to Other Growth-Limiting Factors Such as Nutrients. Bacterial behaviour is controlled by interacting regulatory systems to alter taxis towards energy sources or specific nutrients, $\mathrm{O}_{2}$, and so forth, the uptake of resources, and metabolism to maximise energy production and growth (e.g., [37-39]), and, in P. fluorescens SBW25 populations, diversification and final population sizes are dependent on nutrient levels [20, 40]. However, although $\mathrm{O}_{2}$ levels might be the dominating factor affecting growth, diversification, and fitness in normal static microcosms, we predict that the importance of $\mathrm{O}_{2}$ to 
TABLE 1: Wrinkly Spreader biofilm characteristics in modified microcosms.

\begin{tabular}{lcccc}
\hline Treatment & & $\begin{array}{c}\text { Relative } \\
\text { microcosm growth } \\
\left(G / G_{\mathrm{R}}\right)\end{array}$ & $\begin{array}{c}\text { Relative } \\
\text { biofilm strength } \\
\left(S / S_{\mathrm{R}}\right)\end{array}$ & $\begin{array}{c}\text { Relative } \\
\text { attachment } \\
\left(A / A_{\mathrm{R}}\right)\end{array}$ \\
\hline Standard KB ${ }^{*}$ microcosm & No oil, 1x nutrients & $1.000 \pm 0.051^{\mathrm{a}}$ & $1.000 \pm 0.167^{\mathrm{a}}$ & $1.000 \pm 0.081^{\mathrm{a}}$ \\
\hline \multirow{3}{*}{ With oil overlays } & $10 \mathrm{~mm}$ & $0.451 \pm 0.026^{\mathrm{b}}$ & $0.153 \pm 0.024^{\mathrm{b}}$ & $0.311 \pm 0.026^{\mathrm{b}}$ \\
& $20 \mathrm{~mm}$ & $0.381 \pm 0.017^{\mathrm{b}}$ & $0.226 \pm 0.035^{\mathrm{b}}$ & $0.394 \pm 0.030^{\mathrm{b}}$ \\
\hline With reduced-nutrient levels & $40 \mathrm{~mm}$ & $0.247 \pm 0.011^{\mathrm{c}}$ & $0.194 \pm 0.046^{\mathrm{b}}$ & $0.375 \pm 0.044^{\mathrm{b}}$ \\
\hline Altered A-L interface shapes & $0.1 \mathrm{x}$ & $0.408 \pm 0.009^{\mathrm{b}}$ & $0.194 \pm 0.021^{\mathrm{b}}$ & $0.535 \pm 0.106^{\mathrm{b}}$ \\
& $0.01 \mathrm{x}$ & $0.117 \pm 0.002^{\mathrm{c}}$ & $0.113 \pm 0.016^{\mathrm{b}}$ & $0.343 \pm 0.073^{\mathrm{b}}$ \\
\hline
\end{tabular}

Microcosms were incubated for 3 days before assay. Relative means \pm SE are shown $(n=6)$. The reference microcosms for the oil-overlay and reduced-nutrient assays were provided by the standard $\mathrm{KB}^{*}$ microcosms. The reference microcosms for the altered A-L interface shape assays were provided by the concave microcosms. Comparisons of relative means were by assay and treatment. For the oil-overlay and reduced-nutrient experiments, means not linked by the same letter are significantly different (TK-HSD, $\alpha=0.05)$. For the A-L interface shape experiment, means which are significantly different are indicated by $*(t$-test, $p \leq 0.05)$.

WS fitness will decrease as other factors become progressively growth-limiting, and we have used microcosms with reduced-nutrient levels to test this prediction.

Preliminary tests were used to establish the notion that P. fluorescens SBW25 could grow in microcosms containing $0.1 \mathrm{x}$ and $0.01 \mathrm{x}$ normal nutrient levels, but not at $0.001 \mathrm{x}$ (Dunnett's test with control, $\alpha=0.05$ ). This is in agreement with other studies in which the impact of reduced-nutrient levels on the growth rate of P. fluorescens SBW25, carrying capacity, and time lag under similar conditions has been investigated [40]. Further tests showed that WS biofilms were produced under these conditions, but, like those in the oil-overlay experiments, they were significantly reduced in terms of growth, strength, and attachment levels compared to the reference microcosms containing normal nutrient levels (Table 1). Similarly, a large significant decrease in relative fitness to $0.096 \pm 0.019\left(W / W_{\mathrm{R}}\right)$ was observed in reducednutrient microcosms compared to the reference microcosms with normal nutrient levels (TK-HSD, $\alpha=0.05$ ) (Figure 4). This confirms that WS fitness is sensitive to nutrient levels but, more specifically, indicates that the effect of $\mathrm{O}_{2}$ on WS fitness depends on the availability of other resources which might become growth-limiting, revealing more complexity in this simple model system. From other work, both absolute nutrient levels and complexity are known to affect the adaptive radiation of $P$. fluorescens SBW25 populations, with reduced levels and single nutrient sources resulting in a lower frequency of Wrinkly Spreaders [19, 20, 40]. We can confirm this as, under the conditions used here, $\sim 60 \%$ of the cells sampled after three days from microcosms with normal nutrient levels inoculated with wild-type $P$. fluorescens SBW25 were Wrinkly Spreaders; in contrast, none were observed in reduced-nutrient microcosms after the same time. However, we have not investigated how changing the inoculum size, growth rates, or final population sizes might also interact with reduced-nutrient levels to affect the appearance of Wrinkly Spreaders in radiating populations of P. fluorescens SBW25.
Collectively, the oil-overlay and reduced-nutrient experiments demonstrate the hidden complexity in this seemingly simple model system where WS fitness is affected predominantly by $\mathrm{O}_{2}$ levels, but also by $\mathrm{O}_{2}$ and nutrient interactions. We predict that, under sufficiently low $\mathrm{O}_{2}$ conditions, nutrient levels would begin to dominate, and, under optimal $\mathrm{O}_{2}$ and nutrient conditions, the physical dimensions of the microcosm would become important with large SA/V ratios favouring the Wrinkly Spreader and low ratios possibly selecting for low- $\mathrm{O}_{2}$-adapted mutants. Clearly, any response to altered resource levels or physical dimensions will be sensitive to the cell densities and relative numbers of Wrinkly Spreaders and non-biofilm-forming competitors used for inoculation as well as the length of incubation.

\subsection{Concave A-L Interfaces and the Importance of the Meniscus} Trap. The meniscus trap is a feature of the physicality of microcosms which we speculate aids WS biofilm formation and fitness. Although the progression of WS biofilm formation has yet to be recorded, it is likely that individual cells or rafts of cells first attach to the vial walls at the meniscus before growing outwards to cover the A-L interface. We expect that the acute angle formed at the meniscus by the air-liquid-solid surface (A-L-S) interface will form a high- $\mathrm{O}_{2}$ trap for cells (Figure 5) which might be recruited to the A-L interface by swimming motility, bioconvection cells, and penetration of the interface by a combination of cellulose, attachment factor, and surfactant expression [16, 23, 41-43]. This model is based on sessile drops where a hydrodynamic vortex is created by bacterial $\mathrm{O}_{2}$ taxis and the downward gravitational effect due to cell density which enhances $\mathrm{O}_{2}$ diffusion into the liquid and traps cells near the A-L-S interface [43].

We overfilled microcosms to produce convex-shaped A-L interfaces lacking meniscus traps to investigate the impact this would have on WS biofilm formation and fitness (Figure 5). Preliminary experiments showed that WS biofilms formed in convex microcosms, though they were reduced in terms of growth, strength, and attachment levels 


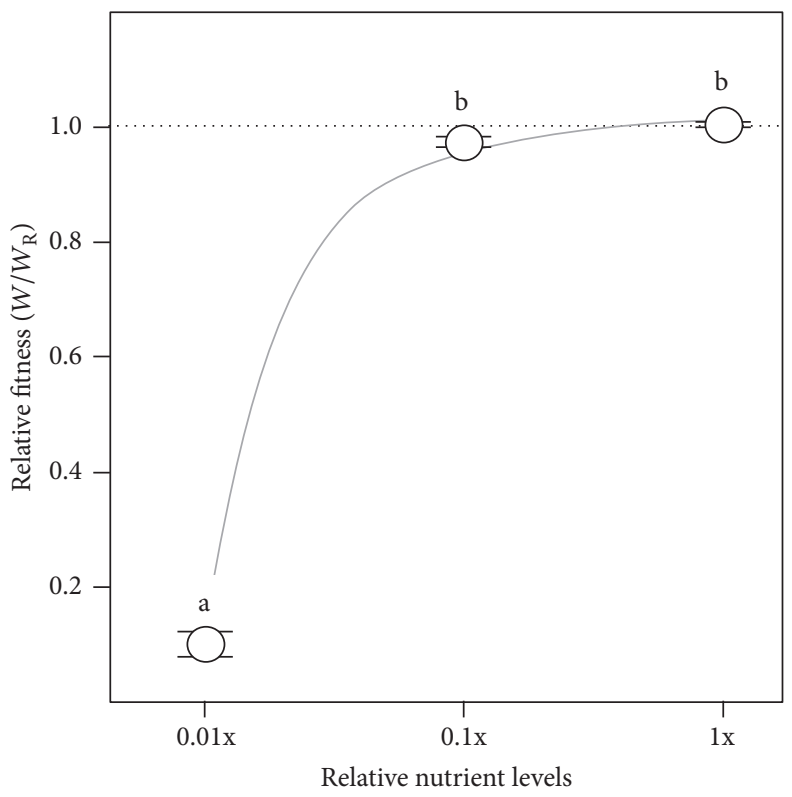

FIGURE 4: Reducing the importance of $\mathrm{O}_{2}$ reduces Wrinkly Spreader fitness. Nutrient levels were manipulated to reduce the importance of $\mathrm{O}_{2}$ in static microcosms. The nutrient component in $\mathrm{KB}^{*}$ was diluted to produce microcosms with $0.01 \mathrm{x}$ and $0.1 \mathrm{x}$ normal nutrient levels. Microcosms were incubated for 3 days before assay and the relative competitive fitness of the Wrinkly Spreader was determined compared to the non-biofilm-forming P. fluorescens SBW25. Here, the relative fitness $\left(W / W_{\mathrm{R}}\right)$ is provided where the reference microcosms with normal (1x) nutrient levels have relative fitness of one (marked by the horizontal dashed line); the curve indicated here is illustrative only. Means \pm $\mathrm{SE}$ are shown $(n=6)$, and means not linked by the same letter are significantly different (TK-HSD, $\alpha=0.05)$. The standard KB* microcosm data are also shown in Figure 2.

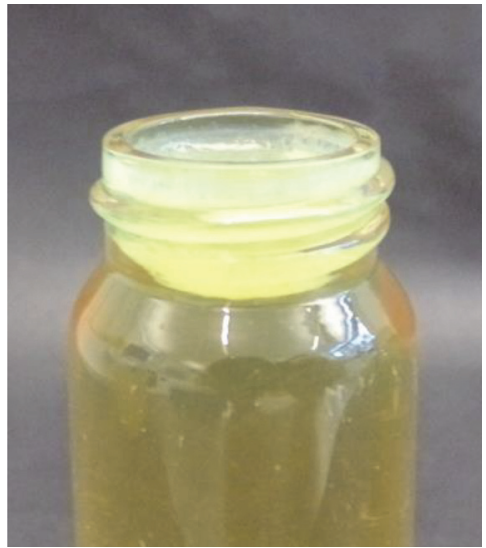

(a)

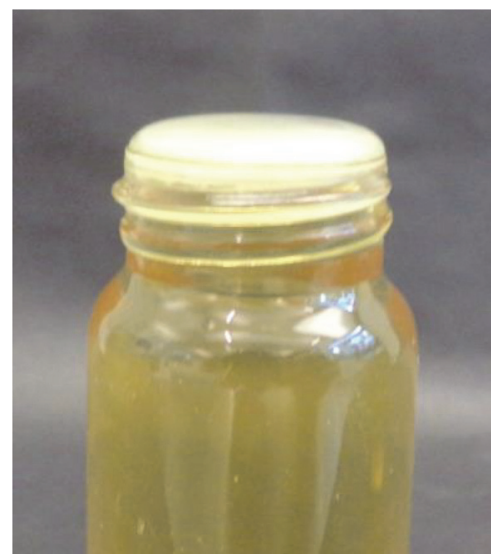

(b)

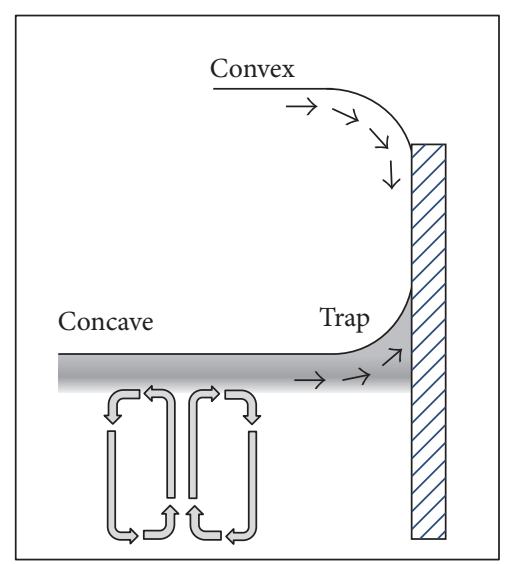

(c)

FIgURE 5: Wrinkly Spreaders colonise concave- and convex-shaped A-L interfaces. Microcosm vials were filled to the brim with KB ${ }^{*}$ to produce (a) concave- and (b) convex-shaped A-L interfaces. A schematic of the upper regions of a microcosm (c) shows how the concaveshaped A-L interface interacts with the vial walls to produce the meniscus trap (which does not form with convex-shaped A-L interfaces). Only the top layer of liquid will have high- $\mathrm{O}_{2}$ levels (grey), and bacteria near the A-L interface are likely to be displaced radially towards the meniscus trap (black arrows) driven in part by bioconvection cells produced by bacterial motility.

compared to the reference microcosms with normal concave A-L interfaces (Table 1). Although relative microcosm growth and biofilm strengths increased similarly in concave and convex microcosms over three days of incubation, relative attachment levels in convex microcosms were found to be significantly reduced by $\sim 0.6 \mathrm{x}$ compared to concave microcosms after three days (TK-HSD, $\alpha=0.05$ ) (Figure 6). This suggests that the meniscus trap supports better growth of attached cells and the early establishment of the WS biofilm in concave microcosms. A corresponding small but significant reduction in relative fitness to $0.959 \pm 0.004\left(W / W_{R}\right)$ was found in convex microcosms compared to concave microcosms after one day (Figure 7), but not after the third day at which point relative fitness had dropped to $\sim 0.9$ 


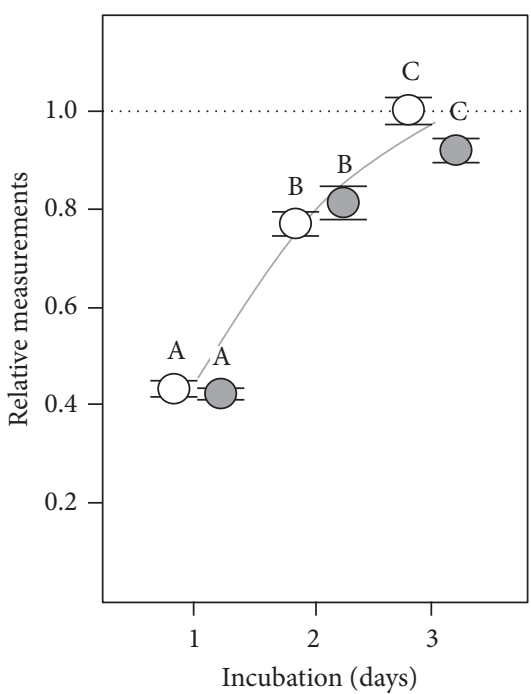

(a) Growth

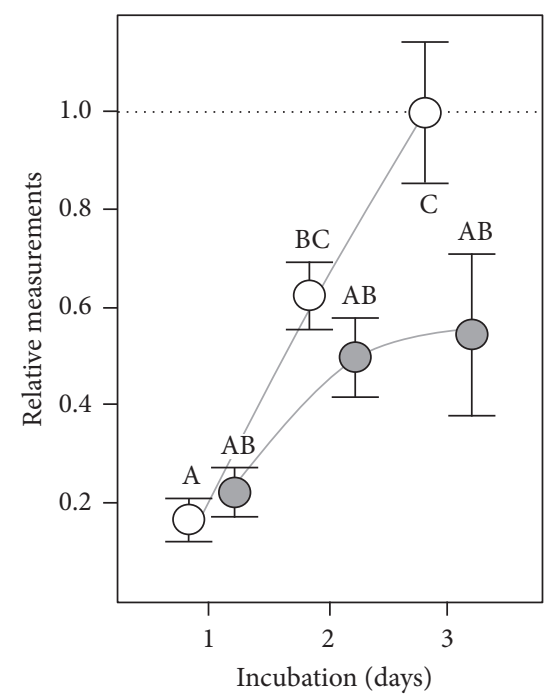

(b) Strength

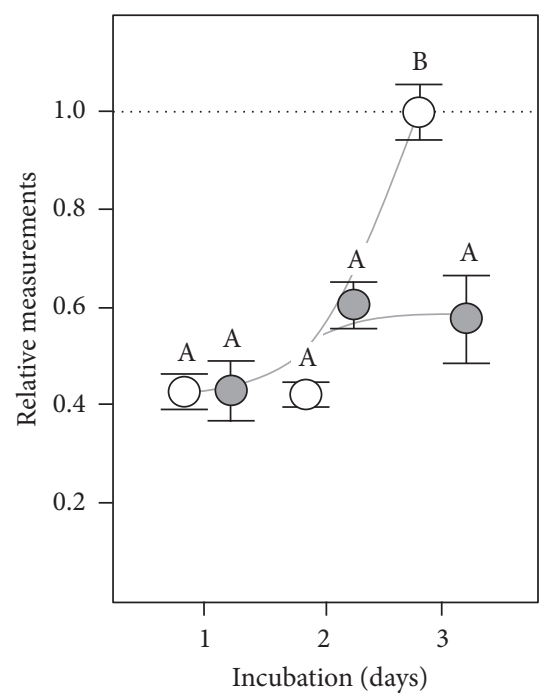

(c) Attachment

FiguRE 6: WS biofilm characteristics differed slightly between concave- and convex-shaped A-L interfaces. Microcosms with concave(normal) and convex-shaped A-L interfaces were used to assess WS biofilm characteristics. $\mathrm{KB}^{*}$ microcosms were incubated for 1, 2, or 3 days before assay. Relative measurements are shown for microcosm growth ((a), $\left.G / G_{\mathrm{R}}\right)$, WS biofilm strength $\left((\mathrm{b}), S / S_{\mathrm{R}}\right)$, and attachment levels $\left((\mathrm{c}), A / A_{\mathrm{R}}\right)$ where the 3 -day-old concave reference microcosms have a value of one (marked by the horizontal dashed line); the curves indicated are illustrative only. Means \pm SE are shown $(n=6)$ and means not linked by the same letter are significantly different (TK-HSD, $\alpha=0.05)$.

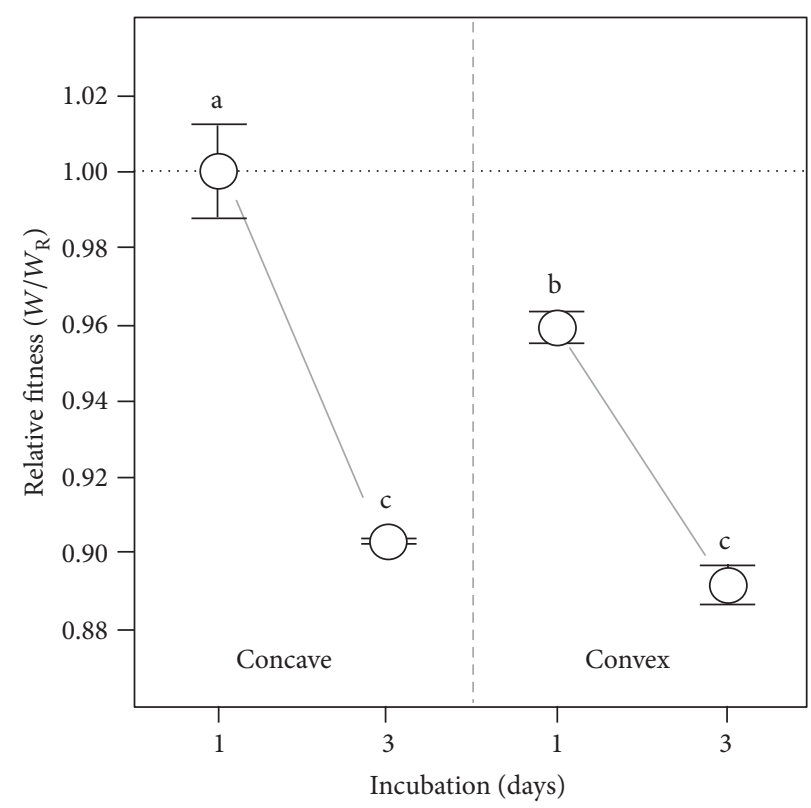

Figure 7: Concave A-L interface favours Wrinkly Spreader fitness. Microcosms with concave- (normal) and convex-shaped A-L interfaces were used to assess the importance of the meniscus trap in WS fitness. Microcosms were incubated for 1 or 3 days before assay and the relative competitive fitness of the Wrinkly Spreader was determined compared to the non-biofilm-forming P. fluorescens SBW25. Here, the relative fitness $\left(W / W_{\mathrm{R}}\right)$ is provided where the reference microcosms with a concave-shaped A-L interface have relative fitness of one (marked by the horizontal dashed line); the curve indicated here is illustrative only. Means \pm SE are shown $(n=6)$ and means not linked by the same letter are significantly different (TK-HSD, $\alpha=0.05$ ). 
$\left(W / W_{\mathrm{R}}\right)$ in both types of microcosm (TK-HSD, $\left.\alpha=0.05\right)$, perhaps as the result of the unintentional damage of the biofilms caused by the daily additions of media required to maintain A-L interface shapes.

\section{Conclusion}

Experimental populations of $P$. fluorescens SBW25 in simple microcosms have proved to be a useful model system for investigating bacterial adaptive radiation and allowed mechanistic links to be made between mutation, the Wrinkly Spreader morphotype, and WS fitness advantage. Although this system is simple, our results provide new insight into the effects of several environmental parameters on the relative fitness of this numerically dominant class of evolved niche specialist. As such, the work represents an advance in our understanding of this influential model system, highlighting the way in which $\mathrm{O}_{2}$ and other factors interact, increasing complexity, and impacting WS fitness. Further manipulation of the system to enhance the development of low- $\mathrm{O}_{2}$ adaptive morphotypes colonising the liquid column below the WS biofilm would allow a more comprehensive understanding of the adaptive radiation of an ancestral genotype into interlinked niches with quite different selective pressures.

\section{Competing Interests}

The authors declare that there are no competing interests regarding the publication of this paper.

\section{Acknowledgments}

The authors thank Anna Koza who helped conduct the preliminary experiments that formed the basis for this work. Anna Kuśmierska's visit to Abertay was supported through the IAESTE Student Exchange Programme and by Abertay University, and the authors further appreciated the support provided to her by the Graduate School. Andrew Spiers is also Member of the Scottish Alliance for Geoscience Environment and Society (SAGES).

\section{References}

[1] M. Kirschner and J. Gerhart, "Evolvability," Proceedings of the National Academy of Sciences of the United States of America, vol. 95 , no. 15 , pp. 8420-8427, 1998.

[2] D. Schulter, The Ecology of Adaptive Radiation, Oxford Series in Ecology and Evolution, OUP, Oxford, UK, 2000.

[3] J. Odling-Smee, D. H. Erwin, E. P. Palkovacs, M. W. Feldman, and K. N. Laland, "Niche construction theory: a practical guide for ecologists," The Quarterly Review of Biology, vol. 88, no. 1, pp. $4-28,2013$.

[4] J. N. Thompson, Relentless Evolution, University of Chicago Press, Chicago, Ill, USA, 2013.

[5] R. C. MacLean, "Adaptive radiation in microbial microcosms," Journal of Evolutionary Biology, vol. 18, no. 6, pp. 1376-1386, 2005.

[6] A. Buckling, R. Craig MacLean, M. A. Brockhurst, and N. Colegrave, "The Beagle in a bottle," Nature, vol. 457, no. 7231, pp. 824-829, 2009.
[7] A. J. Spiers, "Bacterial evolution in simple microcosms," in Microcosms: Ecology, Biological Implications and Environmental Impact, C. H. Harris, Ed., Microbiology Research Advances Series C., pp. 107-127, Nova, Hauppauge, NY, USA, 2013.

[8] H. P. Steenackers, I. Parijs, K. R. Foster, J. Vanderleyden, and E. Banin, "Experimental evolution in biofilm populations," FEMS Microbiology Reviews, vol. 40, no. 3, pp. 373-397, 2016.

[9] P. B. Rainey and M. Travisano, "Adaptive radiation in a heterogeneous environment," Nature, vol. 394, no. 6688, pp. 69-72, 1998.

[10] O. V. Moshynets and A. J. Spiers, "Viewing biofilms within the larger context of bacterial aggregations," in Microbial BiofilmsImportance and Applications, D. Dhanasekaran and N. Thajuddin, Eds., pp. 3-22, InTech Publishers, Rijeka, Croatia, 2016.

[11] A. Koza, O. Moshynets, W. Otten, and A. J. Spiers, "Environmental modification and niche construction: developing $\mathrm{O}_{2}$ gradients drive the evolution of the Wrinkly Spreader," International Society of Microbial Ecology Journal, vol. 5, no. 4, pp. 665-673, 2011.

[12] J. B. Yoder, E. Clancey, S. Des Roches et al., "Ecological opportunity and the origin of adaptive radiations," Journal of Evolutionary Biology, vol. 23, no. 8, pp. 1581-1596, 2010.

[13] R. C. E. Flohr, C. J. Blom, P. B. Rainey, and H. J. E. Beaumont, "Founder niche constrains evolutionary adaptive radiation," Proceedings of the National Academy of Sciences of the United States of America, vol. 110, no. 51, pp. 20663-20668, 2013.

[14] A. J. Spiers, S. G. Kahn, M. Travisano, J. Bohannon, and P. B. Rainey, "Adaptive divergence in Pseudomonas fluorescens. 1. Determinants of wrinkly spreader fitness and the cause of an evolutionary transition," Genetics, vol. 161, no. 1, pp. 33-46, 2002.

[15] A. J. Spiers, J. Bohannon, S. M. Gehrig, and P. B. Rainey, "Biofilm formation at the air-liquid interface by the Pseudomonas fluorescens SBW25 wrinkly spreader requires an acetylated form of cellulose," Molecular Microbiology, vol. 50, no. 1, pp. 15-27, 2003.

[16] A. J. Spiers and P. B. Rainey, "The Pseudomonas fluorescens SBW25 wrinkly spreader biofilm requires attachment factor, cellulose fibre and LPS interactions to maintain strength and integrity," Microbiology, vol. 151, no. 9, pp. 2829-2839, 2005.

[17] E. Bantinaki, R. Kassen, C. G. Knight, Z. Robinson, A. J. Spiers, and P. B. Rainey, "Adaptive divergence in experimental populations of Pseudomonas fluorescens. III. Mutational origins of wrinkly spreader diversity," Genetics, vol. 176, no. 1, pp. 441-453, 2007.

[18] A. J. Spiers, "A mechanistic explanation linking adaptive mutation, niche change, and fitness advantage for the wrinkly spreader," International Journal of Evolutionary Biology, vol. 2014, Article ID 675432, 10 pages, 2014.

[19] M. Travisano and P. B. Rainey, "Studies of adaptive radiation using model microbial systems," American Naturalist, vol. 156, no. 4, pp. S35-S44, 2000.

[20] R. Kassen, M. Llewellyn, and P. B. Rainey, "Ecological constraints on diversification in a model adaptive radiation," Nature, vol. 431, no. 7011, pp. 984-988, 2004.

[21] J. H. Green, A. Koza, O. Moshynets, R. Pajor, M. R. Ritchie, and A. J. Spiers, "Evolution in a test-tube: rise of the wrinkly spreaders," Journal of Biological Education, vol. 45, no. 1, pp. 54-59, 2011.

[22] W. E. Huang, S. Ude, and A. J. Spiers, "Pseudomonas fluorescens SBW25 biofilm and planktonic cells have differentiable Raman spectral profiles," Microbial Ecology, vol. 53, no. 3, pp. 471-474, 2007. 
[23] A. Koza, P. D. Hallett, C. D. Moon, and A. J. Spiers, "Characterization of a novel air-liquid interface biofilm of Pseudomonas fluorescens SBW25," Microbiology, vol. 155, no. 5, pp. 1397-1406, 2009.

[24] K. Yamamoto, H. Arai, M. Ishii, and Y. Igarashi, "Trade-off between oxygenand iron acquisition in bacterial cells at the airliquid interface," FEMS Microbiology Ecology, vol. 77, no. 1, pp. 83-94, 2011.

[25] J. B. Xavier and K. R. Foster, "Cooperation and conflict in microbial biofilms," Proceedings of the National Academy of Sciences of the United States of America, vol. 104, no. 3, pp. 876-881, 2007.

[26] A. J. Spiers, "Wrinkly-Spreader fitness in the two-dimensional agar plate microcosm: maladaptation, compensation and ecological success," PLoS ONE, vol. 2, no. 8, article e740, 2007.

[27] P. B. Rainey and M. J. Bailey, "Physical and genetic map of the Pseudomonas fluorescens SBW25 chromosome," Molecular Microbiology, vol. 19, no. 3, pp. 521-533, 1996.

[28] R. E. Lenski, M. R. Rose, S. C. Simpson, and S. C. Tadler, "Longterm experimental evolution in Escherichia coli. I. Adaptation and divergence during 2000 generations," The American Naturalist, vol. 138, no. 6, pp. 1315-1341, 1991.

[29] M. Robertson, S. M. Hapca, O. Moshynets, and A. J. Spiers, "Air-liquid interface biofilm formation by psychrotrophic pseudomonads recovered from spoilt meat," Antonie van Leeuwenhoek, International Journal of General and Molecular Microbiology, vol. 103, no. 1, pp. 251-259, 2013.

[30] S. Ude, D. L. Arnold, C. D. Moon, T. Timms-Wilson, and A. J. Spiers, "Biofilm formation and cellulose expression among diverse environmental Pseudomonas isolates," Environmental Microbiology, vol. 8, no. 11, pp. 1997-2011, 2006.

[31] R. C. MacLean, G. Bell, and P. B. Rainey, "The evolution of a pleiotropic fitness tradeoff in Pseudomonas fluorescens," Proceedings of the National Academy of Sciences of the United States of America, vol. 101, no. 21, pp. 8072-8077, 2004.

[32] M. J. McDonald, S. M. Gehrig, P. L. Meintjes, X.-X. Zhang, and P. B. Rainey, "Adaptive divergence in experimental populations of Pseudomonas fluorescens. IV. Genetic constraints guide evolutionary trajectories in a parallel adaptive radiation," Genetics, vol. 183, no. 3, pp. 1041-1053, 2009.

[33] Y. C. Udall, Y. Deeni, S. M. Hapca, D. Raikes, and A. J. Spiers, "The evolution of biofilm-forming Wrinkly Spreaders in static microcosms and drip-fed columns selects for subtle differences in wrinkleality and fitness," FEMS Microbiology Ecology, vol. 91, no. 6, Article ID fiv057, 2015.

[34] G. C. Ferguson, F. Bertels, and P. B. Rainey, "Adaptive divergence in experimental populations of Pseudomonas fluorescens. V. insight into the niche specialist fuzzy spreader compels revision of the model Pseudomonas radiation," Genetics, vol. 195, no. 4, pp. 1319-1335, 2013.

[35] P. G. Verschuren, T. D. Cardona, M. J. R. Nout, K. D. De Gooijer, and J. C. Van Den Heuvel, "Location and limitation of cellulose production by Acetobacter xylinum established from oxygen profiles," Journal of Bioscience and Bioengineering, vol. 89, no. 5, pp. 414-419, 2000.

[36] F. D. Gunstone and F. B. Padley, Lipid Technologies and Applications, CRC Press, 1997.

[37] B. L. Taylor, I. B. Zhulin, and M. S. Johnson, "Aerotaxis and other energy-sensing behavior in bacteria," Annual Review of Microbiology, vol. 53, pp. 103-128, 1999.

[38] G. Alexandre, "Coupling metabolism and chemotaxis-dependent behaviours by energy taxis receptors," Microbiology, vol. 156, no. 8, pp. 2283-2293, 2010.
[39] V. Chubukov, L. Gerosa, K. Kochanowski, and U. Sauer, "Coordination of microbial metabolism," Nature Reviews Microbiology, vol. 12, no. 5, pp. 327-340, 2014.

[40] D. W. Armitage, "Experimental evidence for a time-integrated effect of productivity on diversity," Ecology Letters, vol. 18, no. 11, pp. 1216-1225, 2015.

[41] A. Czirók, I. M. Jánosi, and J. O. Kessler, "Bioconvective dynamics: dependence on organism behaviour," The Journal of Experimental Biology, vol. 203, no. 21, pp. 3345-3354, 2000.

[42] J. O. Kessler, G. D. Burnett, and K. E. Remick, "Mutual dynamics of swimming microorganisms and their fluid habitat," in Nonlinear Science at the Dawn of the 21st Century, P. L. Christiansen, M. P. Sorensen, and A. C. Scott, Eds., pp. 409-426, Springer, New York, NY, USA, 2000.

[43] I. Tuval, L. Cisneros, C. Dombrowski, C. W. Wolgemuth, J. O. Kessler, and R. E. Goldstein, "Bacterial swimming and oxygen transport near contact lines," Proceedings of the National Academy of Sciences of the United States of America, vol. 102, no. 7, pp. 2277-2282, 2005. 

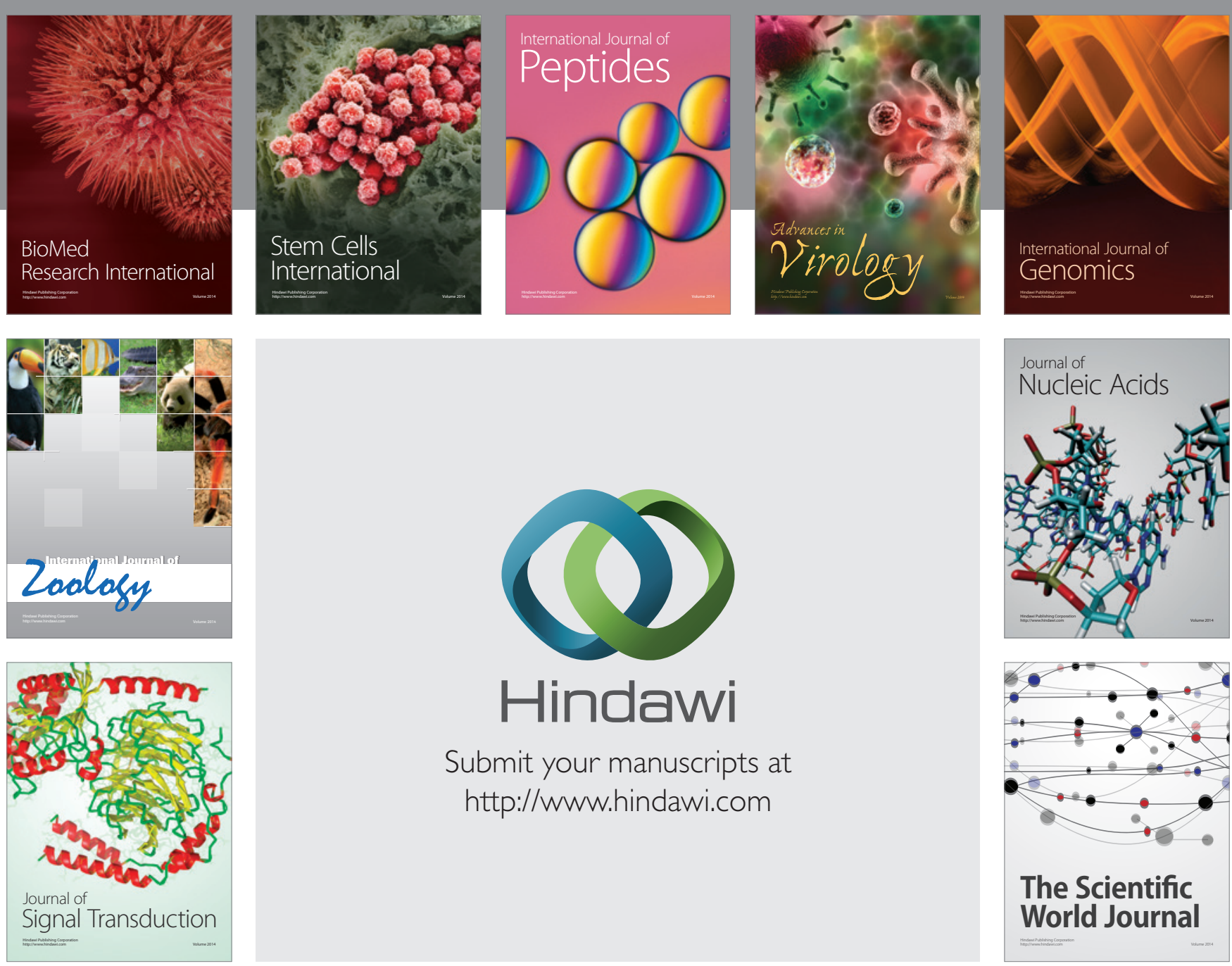

Submit your manuscripts at

http://www.hindawi.com
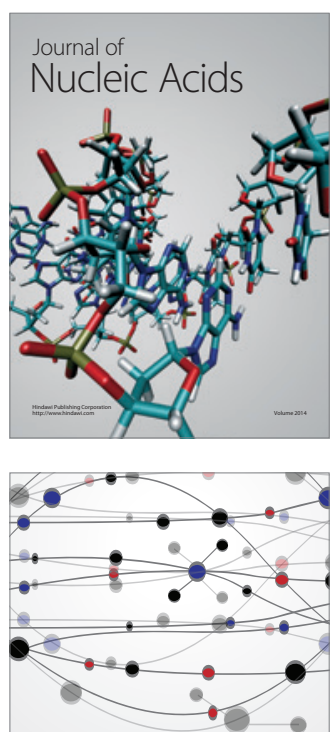

The Scientific World Journal
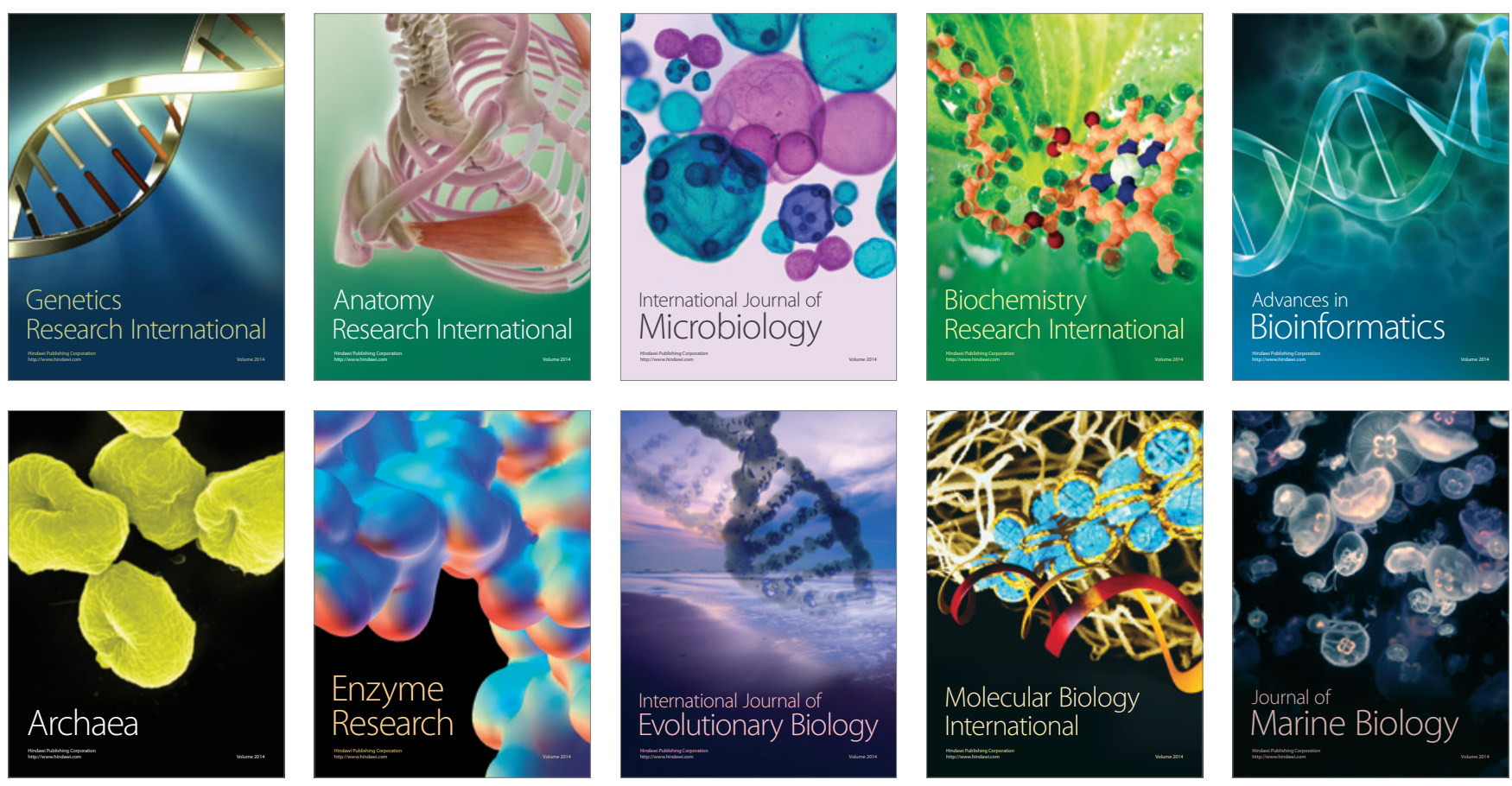